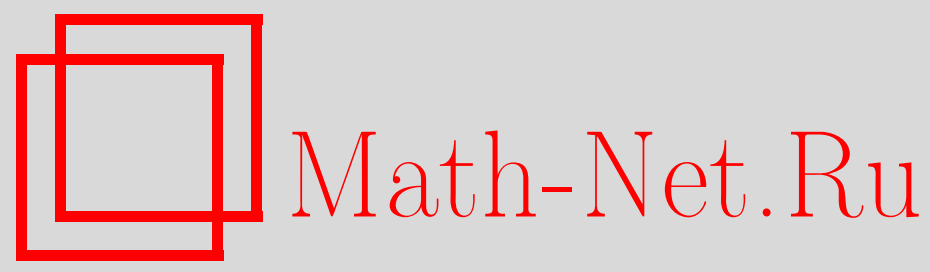

Ф. М. Малышев, В. Е. Тараканов, Обобщенные графы де Брейна, Матем. заметки, 1997, том 62, выпуск 4, 540-548

DOI: https://doi.org/10.4213/mzm1637

Использование Общероссийского математического портала Math-Net.Ru подразумевает, что вы прочитали и согласны с пользовательским соглашением http://www.mathnet.ru/rus/agreement

Параметры загрузки:

IP: 3.93 .64 .190

26 апреля 2023 г., 03:35:10

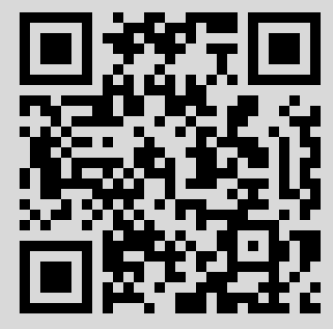




\title{
ОБОБЩЕННЫЕ ГРАФЫ ДЕ БРЕЙНА
}

\author{
Ф. М. Малышев, В.Е. Тараканов
}

Изучаются ориентированные графы, в которых для любой пары вершин существует единственный путь из одной вершшиы в другую заданной длины, не зависящей от конкретно выбранной пары вершин. Этот класс графов является естественным обобщением широко распространенных графов де Брейна и сохраняет их наиболее существенные свойства. Получен ряд результатов о структуре и методах построения таких графов.

Библиография: 7 названий.

Графы де Брейна благодаря своему совершенству и широте их применения занимают особое место среди специальных классов ориентированных графов (см., например, [1]-[5]). В данной работе представлен класс графов, в которых сохраняются наиболее важные качества графов де Брейна. Называем их обобщенными графами де Брейна или, кратко, Ә-графами.

Мы рассматриваем лишь ориентированные графы (орграфы), поэтому, говоря о графе, всегда имеем в виду орграф. Используемую терминологию и некоторые результаты, относящиеся к графам, можно найти в [6], а специально к графам де Брейна - в [1].

ОПРЕДЕЛЕНИЕ 1 . Пусть $n, r$ - натуральные числа, $n>1, r \geqslant 1$. Ориентированньй граф на $n$ вершинах назьвается $\partial$-графом порядка $r$, если для любой пары его вершин существует единственный направленньй путь длины $r$ из одной вершины в другую.

При $r=1 \partial$-граф - это полньй граф на $n$ вершинах. Примеры $\partial$-графов при $r>1$

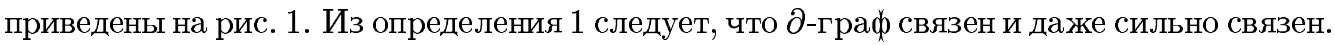
Ясно также, что в $\partial$-графе не может быть двух различных направленных путей длины $l \leqslant r$ с общим началом и концом, в частности, исключены параллельные дуги.

С данньп $\partial$-графом $\Gamma$ порядка $r$ можно связать другие $\partial$-графы. Непосредственно из определения 1 следует, что если в Г изменить направление по каждой его дуге на противоположное, то получающийся граф $\bar{\Gamma}$ есть также $\partial$-граф порядка $r$. Пусть, далее, $\Gamma^{+}-$граф, двойственный $\partial-г$ рафу $\Gamma$ (см. [1]): вершины $\Gamma^{+}$суть дуги $\Gamma$, и в $\Gamma^{+}$есть дуга из вершины $e_{1}$ в вершину $e_{2}$ тогда и только тогда, когда в $Г$ конец дуги $e_{1}$ совпадает с началом дуги $e_{2}$. Понятно, что $\Gamma^{+}$есть $\partial$-граф порядка $r+1$.

Очевидно, граф $\Gamma^{+}$может быть определен и для любого графа $Г$. Он обладает следующим свойством: окрестности любых двух его вершин либо совпадают, либо их пересечение пусто. Это свойство пары вершин графа назовем $\mathscr{N}$-свойством. Как обычно,

Работа второго автора выполнена при финансовой поддержке Российского фонда фундаментальных исследований, грант № 96-01-00531. 
окрестностью $\Gamma(v)$ вершины $v$ графа $Г$ считаем множество концов всех дуг, исходящих из $v$. Если в графе $Г$ для любой пары вершин вьполнено $\mathcal{N}$-свойство, то говорим, что $\Gamma$ обладает $\mathscr{N}$-свойством.

ОПРЕДЕЛЕНИЕ 2. Ә-граф Г порядка $r>1$ назьвается $n p o c m b м$, если он неизоморфен $\Gamma_{1}^{+}$ни для какого $\partial$-графа $\Gamma_{1}$. Полньй граф на $n>1$ вершинах (т.е. $\partial$-граф порядка 1) считаем простым.

Ниже мы докажем, что простые $\partial$-графы порядка $r>1$ исчерпываются $\partial$-графами, имеюшими пару вершин, не обладающую $\mathscr{N}$-свойством.
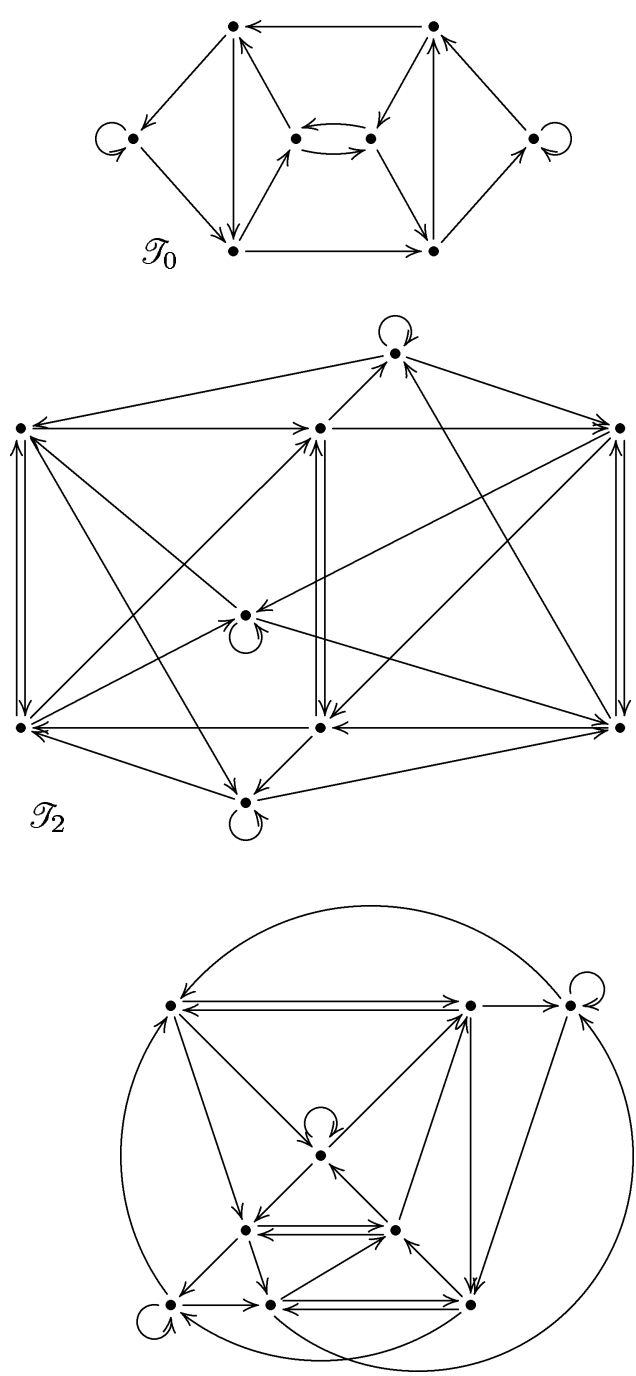

$\mathscr{T}_{4}$
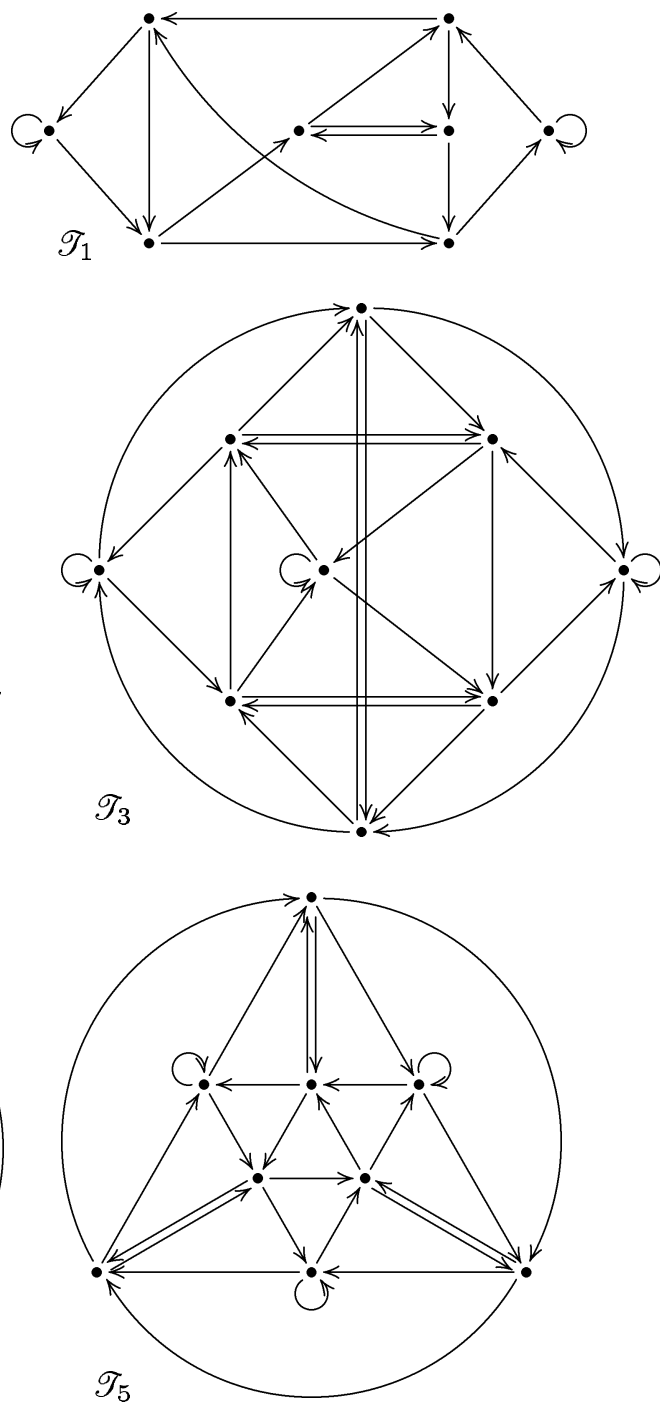

РиС. 1

Приведем примеры $\partial$-графов порядка $r>1$. Основными примерами $\partial$-графов являются графы де Брейна. Пусть $m>1, r>1$ - натуральные числа, $n=m^{r}$. Обо- 
значим через $\mathbb{Z}_{m}$ множество $\{0,1, \ldots, m-1\}$. Множество вершин графа де Брейна порядка $r$ на $n=m^{r}$ вершинах составляют все $r$-мерные целочисленные векторы вида $\left(\nu_{0}, \nu_{1}, \ldots, \nu_{r-1}\right), \nu_{i} \in \mathbb{Z}_{m}, i=\overline{0, r-1}$. Из вершины $\left(\nu_{0}, \nu_{1}, \ldots, \nu_{r-1}\right)$ исходит $m$ дуг в вершины $\left(\varepsilon, \nu_{0}, \ldots, \nu_{r-2}\right)$; в нее входит также $m$ дуг из вершин $\left(\nu_{1}, \ldots, \nu_{r-1}, \varepsilon\right)$, где $\varepsilon-$ любой элемент $\mathbb{Z}_{m}$. Петли имеются в точности на $m$ вершинах $(\nu, \ldots, \nu), \nu \in \mathbb{Z}_{m}$. При $r=1$ граф де Брейна - это полньй граф на $n=m$ вершинах. При $r>1$ граф де Брейна на $m^{r}$ вершинах двойственен графу де Брейна на $m^{r-1}$ вершинах, т.е. он не является простьм. Другиепримеры $\partial$-графов приведены на рис. 1 . Здесь $\mathscr{T}_{0}, \mathscr{T}_{1}-\partial$-графыпорядка 3 на восьми вершинах; $\mathscr{T}_{2}, \mathscr{T}_{3}, \mathscr{T}_{4}, \mathscr{T}_{5}-\partial$-графы порядка 2 на девяти вершинах; $\mathscr{T}_{1}$, $\mathscr{T}_{2}, \mathscr{T}_{3}, \mathscr{T}_{4}-$ простые $\partial$-графы; $\mathscr{T}_{0}$ и $\mathscr{T}_{5}-$ графы де Брейна. Широкие классы простых $\partial$-графов строятся ниже в теореме 3 путем “локальных деформаций” графов де Брейна.

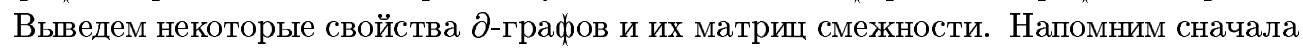
хорошо известные свойства матриц смежности ориентированных графов (см. [6]). Если $A=\left(a_{i j}\right)$ - матрица смежности графа $\Gamma, a_{i j}=0$ или 1 , то число путей длины $l$ из вершины с номером $i$ в вершину с номером $j$ равно элементу $a_{i j}^{(l)}$ в матрице $A^{l}, l=1,2, \ldots$. Число петель в $\Gamma$ - это число единиц на главной диагонали $A$, т.е. оно равно следу матрицы $A$.

ТЕОРема 1. Пусть Г-д-граф порядка $r$. Тогда

а) число п вериин графа Г есть $r$-я степень челого числа $\mathrm{m}$;

б) из кажсдой вериины Г исходит всего $m$ дуг;

в) в кахдую вериину Г входит всего $m$ дуг;

г) число петель в Г равно

ДокАЗАтЕЛьСтво. а) Обозначим через $X=X(\Gamma)$ матрицу смежности $\partial$-графа $Г$. Требование определения 1 равносильно выполнению условия

$$
X^{r}=J
$$

где $J$ - вещественная матрица порядка $n$, все элементы которой равны 1 . Для характеристического многочлена матрицы $J$ имеем представление $\chi_{J}(\lambda)=\lambda^{n}-n \lambda^{n-1}$. Собственные значения $J$ - это $n$ и $(n-1)$-кратный 0 . Поэтому согласно (1) собственные значения $X$ - это $m$ и $(n-1)$-кратный 0 , где $m^{r}=n$, и

$$
\chi_{X}(\lambda)=\lambda^{n}-m \lambda^{n-1} .
$$

Число $m$, будучи следом целочисленной матрицы $X$, должно быть целым.

б) Рассмотрим линейные преобразования вещественного пространства $\mathbb{R}^{n}$, представленные (в естественном базисе) матрицами $J$ и $X$. Вектор-столбец $\mathbf{j}=(1,1, \ldots, 1)^{t}$ является, очевидно, собственным вектором преобразования с матрицей $J$, соответствующим собственному значению $n$. Согласно (1) он является также собственным вектором для преобразования с матрицей $X$, отвечающим единственному ненулевому собственному значению $m$, т.е. $X \mathbf{j}=m \mathbf{j}$. Таким образом, сумма элементов в каждой строке $X$, т.е. число единиц в каждой строке, должно равняться $m$, и мы получаем, что из каждой вершины Г исходит всего $m$ дуг.

в) Транспонированная матрица $X^{t}$ также удовлетворяет (1). Поэтому в соответствии с б) число единиц в каждой строке $X^{t}$, т.е. число единиц в каждом столбце $X$, также равно $m$. Таким образом, в каждую вершину $Г$ входит всего $m$ дуг. 
г) Число петель в Г равно числу единищ на главной диагонали $X$. Из (2) следует, что оно равно $m$.

Свойства $\partial$-графов в теореме 1 выведены из соответствующих свойств их матриц смежности. Вьпишем их в явном виде, так как они будут использованы в дальнейшем при построении $\partial$-графов. Пусть $X=X(\Gamma)$ - матрица смежности $\partial$-графа $Г$ порядка $r$. Тогда

a) порядок $n$ матрицы $X$ есть $r$-я степень целого числа $m$;

б) каждая строка $X$ содержит в точности $m$ единиц;

в) каждый столбец $X$ содержит в точности $m$ единиц;

г) число единиц на главной диагонали $X$ равно $m$.

Далее, мы укажем критерий простоты $\partial$-графа. Он получается как следствие утверждения, относящегося к более широкому классу графов. Пусть $\Gamma$ - связньй ориентированньй граф с множеством вершин $V$. Напомним, что доминируюшим множеством графа Г называется такое подмножество $D \subseteq V$, что

$$
V=\bigcup_{v \in D} \Gamma(v) .
$$

Если при этом никакое собственное подмножество множества $D$ не обладает указанным свойством, то $D$ назьвается минимальным доминирующим множеством.

ТЕОРема 2. Пусть $\Gamma=(V, E)$ - связный ориентированный граф смножеством вериин $V$ и мнохеством дуг Е. Предполохим, что выполнены условия:

а) Г обладает $\mathscr{N}$-свойством;

б) если $v_{1}, v_{2} \in V$ и в Г есть путь длины 2 из $v_{1}$ в $v_{2}$, то такой путь единственный.

Тогда существует и единственен с точностью до изоморфизма связный ориентированныци граф $\Gamma_{1}$, для которого $\Gamma_{1}^{+} \cong \Gamma$. Если при этом $\Gamma-\partial$-граф порядка $r>1$, то $\Gamma_{1}-\partial$-граф порядка $r-1$.

ДокАЗАТЕЛЬСТво. Пусть $D$ - какое-либо минимальное доминирующее множество для $\Gamma$. Так как $\Gamma$-граф с $\mathcal{N}$-свойством, то для любых двух различных вершин $v_{1}, v_{2} \in D$ имеем $\Gamma\left(v_{1}\right) \cap \Gamma\left(v_{2}\right)=\varnothing$ (иначе $D$ не было бы минимальным). Поэтому любой вершине $w \in V$ однозначно соответствует вершина $v \in D$ такая, что $w \in \Gamma(v)$. Определим теперь графф $\Gamma^{-}=\left(V^{-}, E^{-}\right)$с множеством вершин $V^{-}$и множеством дуг $E^{-}$. Пусть множество вершин $V^{-}$графа $\Gamma^{-}$составляют все вершины какого-либо минимального доминирующего множества $D$ графа $\Gamma$. Если $v_{1}, v_{2} \in D$, то пусть $\left(v_{1}, v_{2}\right) \in E^{-}$тогда и только тогда, когда найдутся две вершины $w_{1} \in \Gamma\left(v_{1}\right), w_{2} \in \Gamma\left(v_{2}\right)$ такие, что $\left(w_{1}, w_{2}\right) \in E$. Заметим, что если $w, w^{\prime} \in \Gamma(v), v \in V$, то при $w \neq w^{\prime}$ имеем $\Gamma(w) \cap \Gamma\left(w^{\prime}\right)=\varnothing$, иначе в $Г$ было бы вопреки условию теоремы два пути длины 2 из $v$ в вершину из этого пересечения. Поэтому если $\left(v_{1}, v_{2}\right) \in E^{-}$, то соответствуюшая вершина $w_{1} \in \Gamma\left(v_{1}\right)$ определяется однозначно. С другой стороны, в качестве $w_{2}$ можно брать любую вершину в $\Gamma\left(v_{2}\right)$, так как в силу $\mathscr{N}$-свойства

$$
\Gamma\left(w_{1}\right)=\Gamma\left(v_{2}\right)
$$

Построим теперь изоморфизм $\varphi$ графов $\left(\Gamma^{-}\right)^{+}$и $Г$. Пусть $e=\left(v_{1}, v_{2}\right) \in E^{-}$, где $v_{1}, v_{2} \in V^{-}=D$. Положим $\varphi\left(\left(v_{1}, v_{2}\right)\right)=w_{1}$, где $w_{1} \in \Gamma\left(v_{1}\right)$ - вершина, однозначно, как показано выше, определяемая дугой $e$ из условия (3). Обратно, если $w_{1} \in V$, то 
$w_{1}$ можно сопоставить дугу $\left(v_{1}, v_{2}\right) \in E^{-}$так, что вершины $v_{1}, v_{2} \in D$ однозначно определяются условиями $w_{1} \in \Gamma\left(v_{1}\right)$ и $(3)$. Таким образом, соответствие $\varphi$ взаимно однозначно. Далее, очевидно, что $\left(e_{1}, e_{2}\right)-$ дуга в $\left(\Gamma^{-}\right)^{+}\left(\right.$т.е. $\left.e_{1}=\left(v_{1}, v_{2}\right), e_{2}=\left(v_{2}, v_{3}\right)\right)$ тогда и только тогда, когда $\left(\varphi\left(e_{1}\right), \varphi\left(e_{2}\right)\right) \in E$. Таким образом, $\varphi-$ изоморфизм $\left(\Gamma^{-}\right)^{+}$ и Г.

Пусть теперь $\Gamma-\partial$-граф порядка $r>1$. Ясно, что если $v_{1}, v_{2}-$ две вершины из $V^{-}=D$, то в $\Gamma^{-}$существует единственньй путь длины $r-1$, соответствующий единственному пути длины $r$ из $\varphi\left(\left(u_{1}, v_{1}\right)\right)$ в $\varphi\left(\left(v_{2}, u_{2}\right)\right)$, где $\left(u_{1}, v_{1}\right),\left(v_{2}, u_{2}\right) \in E^{-}$. Таким образом, $\Gamma^{-}-\partial$-граф порядка $r-1$.

Наконец, если $\Gamma_{1}, \Gamma_{2}$ - два связных ориентированных графа, для которых $\Gamma_{1}^{+} \cong \Gamma_{2}^{+}$, то, очевидно, $\Gamma_{1} \cong \Gamma_{2}$. Теорема доказана.

СлЕДСТВИЕ. Ә-граф Г является простым тогда и только тогда, когда в нем существуют две вершины, окрестности которых не совпадают, но имеют непустое пересечение (т.е. две вершины, которые не обладают $\mathcal{N}$-свойством).

Наряду с представлением $\Gamma \cong\left(\Gamma^{-}\right)^{+}$в теореме 2 для графа $\Gamma$ без параллельных дуг имеем также $\left(\Gamma^{+}\right)^{-} \cong \Gamma$. Действительно, обозначим $\Gamma_{1}=\Gamma^{+}$. Тогда $\Gamma_{1}-г$ раф, обладающий $\mathcal{N}$-свойством, в котором нет двух путей длины 2 с общим началом и концом. По теореме $2 \Gamma^{+}=\Gamma_{1} \cong\left(\Gamma_{1}^{-}\right)^{+}$, ввиду единственности такого представления $\Gamma_{1}$ имеем $\Gamma \cong \Gamma_{1}^{-}=\left(\Gamma^{+}\right)^{-}$. Очевидно также, что $(\bar{\Gamma})^{+}={\overline{\Gamma^{+}}}_{\text {и }}(\bar{\Gamma})^{-}=\overline{\Gamma^{-}}$.

В оставшейся части работы предлагается один метод построения простых $\partial$-графов порядка $r$ на $n=m^{r}$ вершинах для произвольных $m>1$ и $r>1$. При этом за основу берется граф де Брейна на $m^{r}$ вершинах. В нем $m^{2}$ дуг, исходящих из $m$ вершин окрестности $\Gamma(v)$ некоторой вершины $v$, заменяются другими $m^{2}$ дугами; такая процедура может быть повторена еще для нескольких окрестностей.

Далее вершины $\partial$-графа нумеруем числами из множества $\mathbb{Z}_{n}$, понимая их как элементы полной системы наименьших неотрицательных вычетов по модулю $n$. Для числа $\nu \in \mathbb{Z}_{n}, n=m^{r}$, используем представление в $m$-ичной системе счисления:

$$
\nu=\nu_{0}+\nu_{1} m+\cdots+\nu_{r-2} m^{r-2}+\nu_{r-1} m^{r-1}, \quad \nu_{i} \in \mathbb{Z}_{m}, \quad i=\overline{0, r-1} .
$$

Для целого числа $k$ пусть $\bar{k}=k-[k / n] n$, где [·] есть целая часть числа. Ясно, что $\bar{k} \in \mathbb{Z}_{n}$ и $\bar{k} \equiv k \bmod n$. Для данного целого $k$ обозначим через $k^{*}$ число $\overline{k m}$. Ясно, что если $m \mid k$, то $m^{2} \mid k^{*}$.

В графе де Брейна из вершины $\nu \in \mathbb{Z}_{n}$ исходят дуги в вершины $\overline{\nu m}+\varepsilon, \varepsilon \in \mathbb{Z}_{m}$. Пусть $A=A_{m^{r}}$ - матрица смежности графа де Брейна порядка $r$ на $m^{r}$ вершинах; если $m$ и $r$ фиксированы, то назьваем ее кратко матрицей де Брейна. Чтобы более наглядно представить строение матрицы де Брейна, рассмотрим на ней прямоугольную сетку: горизонтали пусть следуют через каждую строку матрицы, а вертикали - через каждые $m$ столбцов. Образовавшиеся прямоугольные ячейки заполнены или сплошь единицами, или только нулями (последние преобладают). Первьй единичньй прямоугольник расположен в левом верхнем углу матрищы $A$. Каждьй следуюший единичньй прямоугольник примыкает своим левьм верхним углом к правому нижнему углу предыдущего единичного прямоугольника. Если очередной прямоугольник оказывается при этом за правой границей $A$, то он как бы передвигается на $n$ позиций влево. Последний единичный прямоугольник расположен в нижнем правом углу матрицы $A$. 
Для аналогичного представления матриц $A^{s}$ при $s>1$ также рассмотрим подобную сетку с вертикалями, следующими через каждые $m^{s}$ столбцов. Единичные прямоугольники снова следуют вниз направо, начиная с левого верхнего угла, соприкасаясь друг с другом вершинами. Это свойство матриц $A^{s}$ легко проверяется индукцией по $s: A^{s}=A \cdot A^{s-1}, s=\overline{2, r}$ (такая проверка облегчает понимание дальнейших рассуждений). Заметим, что матрицы $A^{s}, s=\overline{1, r-1},-$ это $g$-циркулянты при $g=m^{s}$, т.е. такие квадратные матрицы, у которых каждая строка получается из предыдущей циклическим сдвигом на $g$ позищий вправо (см. [7]). По этой причине описанные вьше расположение и способ следования друг за другом ненулевых прямоугольников сетки будем назьвать диагонально-циркулянтным.

В дальнейшем будет использоваться более крупная, в $m$ раз увеличенная, сетка на матрице $A$ с горизонталями, следуюшими через $m$ строк, и вертикалями, следующими через $\mathrm{m}^{2}$ столбцов. Способ следования ненулевых прямоугольных ячеек размера $m \times m^{2}$ в этой сетке такой же, что был описан вьше для мелкой сетки, т.е. диагонально-циркулянтньй: ненулевой прямоугольник соприкасается со следующим за ним своим правьм нижним углом. Нулевые ячейки снова преобладают. Ненулевые ячейки как $(0,1)$-матрицы размера $m \times m^{2}$ все одинаковы. Мы их будем назьвать стандартными матрицами. Положение стандартной подматрицы в $A$ определяется номером ее первой строки $k \in \mathbb{Z}_{n}, m \mid k$. Номером ее первого столбца будет $k^{*}=\overline{m k}$. У следующей за ней стандартной подматрицы в $A$ номер первой строки равен $k+m$, если $k \neq m^{r}-m$.

Предлагаемый нами способ построения $\partial$-графов будет заключаться в построении их матриц смежности путем замены стандартных матриц в нескольких ненулевых ячейках матрицы $A$ на некоторые специальные матрицы, назьваемые допустимыми. $(0,1)$-матрица размера $m \times m^{2}$ назьвается допустимой, если в каждом ее столбце ровно одна единища, а в одной строке не может быть двух единиц, отстоящих одна от другой на расстоянии, кратном $m$. Ясно, что допустимая матрица имеет в точности $m$ единиц в каждой строке, причем номера столбцов, где они расположены, своими младшими цифрами исчерпывают все значения из $\mathbb{Z}_{m}$ (столбцы нумеруются числами из $\mathbb{Z}_{m^{2}}$ в $m$-ичной системе). Если в допустимой матрице есть строка с единицами в столбцах с номерами $\varepsilon m, \varepsilon m+1, \ldots, \varepsilon m+m-1$, где $\varepsilon \in \mathbb{Z}_{m}$, то назьваем ее стандартной $\varepsilon$-строкой. Матрища, получающаяся из стандартной с помощью любой из $m$ ! перестановок еестрок, является допустимой; она имеет стандартную $\varepsilon$-строку для всякого $\varepsilon \in \mathbb{Z}_{m}$.

Теорема 3. Пусть $A=A_{m^{r}}$ - матрица смежнности графа де Брейна на $m^{r}$ вершинах, $m>1, r>1$, с прямоугольной сеткой через $m$ строк $и \mathrm{~m}^{2}$ столбиов, $B_{1}, \ldots, B_{t}-$ некоторый набор из $t \geqslant 1$ допустимых матрич. Пусть $X$ - матрица, получающаяся из $A$ заменой ее ненулевых ячеек с номерами первых строк $k_{i} \in \mathbb{Z}_{n}$, $m \mid k_{i}, i=\overline{1, t}$, на матрицы $B_{i}, i=\overline{1, t}$. Предположим, что при

$$
\varepsilon_{i j}=\frac{k_{i}-k_{j}^{*}}{m} \in \mathbb{Z}_{m}, \quad i, j=\overline{1, t},
$$

допустимая матрица $B_{j}$ имеет стандартную $\varepsilon_{i j}$-строку. Тогда $X$ - матрииа смезности д-графа порядка $r$.

ДокАЗАТЕЛЬСтво. Нам требуется проверить равенство (1). С этой целью будем вычислять матрицы $X^{s}, s=\overline{1, r}$. На матрице $X^{s}, s=\overline{1, r-1}$, рассмотрим сетку с горизонталями, следующими через $m$ строк, и вертикалями, следующими через $m^{s+1}$ столб- 
цов. В результате $X^{s}$ разбивается на $s$-ячейки $x_{i j}^{(s)}$ размера $m \times m^{s+1}, i=\overline{0, m^{r-1}-1}$, $j=\overline{0, m^{r-s-1}-1}$.

Проверим вначале, что ненулевые $s$-ячейки в $X^{s}, s=\overline{1, r-1}$, располагаются в диагонально-циркулянтном порядке, т.е. расположены там же, где и ненулевые $s$-ячейки в матрице $A^{s}$. Для этого воспользуемся индукцией по $s$ и при $s \geqslant 2$ равенством

$$
X^{s}=X^{1} \cdot X^{s-1}
$$

На $X^{s-1}$ рассмотрим дополнительную сетку с горизонталями через $m^{2}$ строк и вертикалями через $m^{s+1}$ столбцов. При этом будем говорить, что матрица $X^{s-1}$ разбивается на $(s-1)$-блоки $\bar{x}_{j k}^{(s-1)}$ размера $m^{2} \times m^{s+1}, j=\overline{0, m^{r-2}-1}, k=\overline{0, m^{r-s-1}-1}$. Из равенства (5) имеем

$$
x_{i k}^{(s)}=\sum_{j=0}^{m^{r-2}-1} x_{i j}^{(1)} \cdot \bar{x}_{j k}^{(s-1)}, \quad i=\overline{0, m^{r-1}-1}, \quad k=\overline{0, m^{r-s-1}-1} .
$$

В этой сумме ненулевым может быть только одно слагаемое, отвечающее при каждом $i \in \overline{0, m^{r-1}-1}$ одной ненулевой 1 -ячейке $x_{i j}^{(1)}$ для некоторого $j \in \overline{0, m^{r-2}-1}, j=j(i)$. Далее, по предположению индукции ненулевые $(s-1)$-блоки $\bar{x}_{j k}^{(s-1)}$ располагаются в диагонально-циркулянтном порядке, а один ненулевой $(s-1)$-блок содержит $m$ ненулевых $(s-1)$-ячеек, располагающихся в $(s-1)$-блоке также в диагонально-циркулянтном порядке. В силу этого при каждом $j \in \overline{0, m^{r-2}-1}$ только при одном $k \in \overline{0, m^{r-s-1}-1}$, $k=k(j),(s-1)$-блок $\bar{x}_{j k}^{(s)}$ ненулевой. Таким образом, при каждом $i \in \overline{0, m^{r-1}-1}$ только при одном $k \in \overline{0, m^{r-s-1}-1}, k=k(j(i)), s$-ячейка $x_{i k}^{(s)}$ ненулевая, и

$$
x_{i, k(j(i))}^{(s)}=x_{i, j(i)}^{(1)} \cdot \bar{x}_{j(i), k(j(i))}^{(s-1)} .
$$

Следуют они одна за другой диагонально-циркулянтньм способом из-за того, что оба семейства сомножителей в (6) этим свойством обладают:

$$
-k\left(j\left((i+1) \bmod m^{r-1}\right)\right)=k\left((j(i)+1) \bmod m^{r-2}\right)=(k(j(i))+1) \bmod m^{r-s-1}
$$

(здесь запись $l \bmod m^{t}$ означает наименьший неотрицательный вычет целого числа $l$ по модулю $\left.m^{t}\right)$.

Докажем теперь три леммы относительно произведений (6). Их формулировки используют понятия $s$-стандартных и $s$-квазистандартных матриц, $s=\overline{1, r-1}$.

Матрицу из нулей и единищ размера $m \times m^{s+1}$, являющуюся ненулевой $s$-ячейкой матрицы $A^{s}$, называем $s$-стандартной. Стандартная матрища - это 1-стандартная матрица. Единицы $s$-стандартной матрицы сосредоточены в $m$ отрезках из $m^{s}$ подряд идущих единиц, располагающихся диагонально-циркулянтньм способом. Матрицу из нулей и единищ размера $m \times m^{s+1}$, у которой в каждом столбце ровно одна единица, называем $s$-квазистандартной. Кроме этого, квазистандартным s-блоком, $s=\overline{1, r-2}$, будем назьвать $(0,1)$-матрицу размера $m^{2} \times m^{s+2}$, в которой имеется только $m$ ненулевых $s$-ячеек (матрищ размера $m \times m^{s+1}$ ), располагающихся в диагонально-циркулянтном порядке и являюшихся $s$-квазистандартными (в частности, $s$-стан-

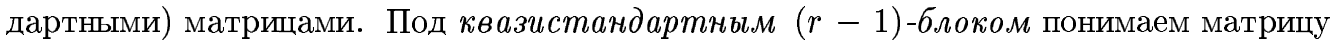
размера $m^{2} \times m^{r}$, состоящую из $m(r-1)$-квазистандартных матриц, располагающихся одна под другой в виде полос ширины $m$, которые нумеруем сверху вниз элементами из $\mathbb{Z}_{m}$. 


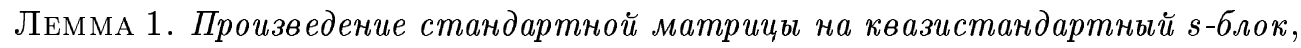

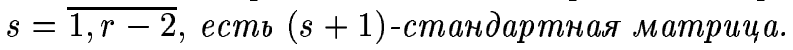

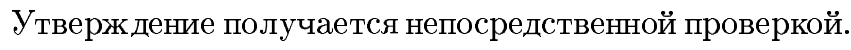

ЛЕмма 2. Произведение 1-квазистандартной (в частности, допустимой) матрицы на квазистандартный $s-б л о к, ~ s=\overline{1, r-2}$, есть $(s+1)$-квазистандартная матрии,

ДокАЗАтЕЛьСтво. Пусть $B=\left(b_{i j}\right)$ - 1-квазистандартная матрища, $C=\left(c_{j k}\right)-$ квазистандартный $s$-блок, $s=\overline{1, r-2}$. Столбец с номером $k$ в $B C$ формируется путем скалярного умножения вектор-строк $0,1, \ldots, m-1$ в $B$ на вектор-столбец $k$ в $C$. Так как $C s$-квазистандартна, имеется в точности один индекс $j=j(k)$, для которого $c_{j k}=1$. Точно так же ввиду квазистандартности $B$ имеется в точности один индекс $i=i(j)$, для которого $b_{i j}=1$. Поэтому умножение строки $i(j)$ из $B$ на столбец $k$ из $C$ дает 1 , а умножение остальных строк дает 0 . Следовательно, в каждом столбце $B C$ имеется в точности одна единища, и, тем самым, матрица $B C$ размера $m \times m^{s+2}$ $(s+1)$-квазистандартна.

ЛЕмма 3. Произведение допустимой (в частности, стандартной) матрицы

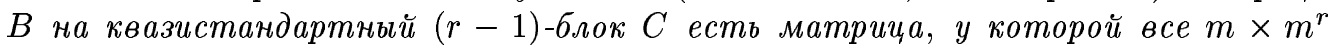
әлементов равны 1 , если выполнено условие: $B$ содерэит стандартную $i$-строку в том случае, когда $i$-я $(r-1)$-квазистандартная подматрииа в $C$ не является $(r-1)$-стандартной, $i \in \mathbb{Z}_{m}$.

ДокАЗАТЕЛЬСтво. Каждую строку матрищы $B$ скалярно умножим на все столбцы матрицы $C$. Если строка стандартная, то фактически происходит скалярное умножение вектор-столбца из $m$ единиц на столбцы $(r-1)$-квазистандартной матрицы, в результате чего всегда получается единица. Если же строка не является стандартной, то ее единицы (после транспонирования строки) могут быть по условию только в областях $(r-1)$-стандартных подматрищ квазистандартного $(r-1)$-блока $C$. Единицы одного столбца этого $(r-1)$-блока, принадлежашие $(r-1)$-стандартньм матрицам, располагаются в строках, у которых младшие разряды номеров одинаковы, а $m$ единиц транспонированной строки допустимой матрицы $B$ расположены в строках, номера которых своими младшими разрядами исчерпывают все значения из $\mathbb{Z}_{m}$. Таким образом, скалярное произведение нестандартной строки $B$ на каждьй столбец $C$ также будет равно 1.

Обратимся, наконец, к индуктивному вычислению матриц $X^{s}, s=\overline{2, r-1}$, с помощью равенств (5) и (6). Из лемм 1 и 2 заключаем, что в матрице $X^{r-1}(r-1)$-ячейки (полосы размера $\left.m \times m^{r}\right)$ с номерами первых строк $k_{i}, i=\overline{1, t}$, будут $(r-1)$-квазистандартными, а все остальные $(r-1)$-ячейки в $X^{r-1}$ будут $(r-1)$-стандартными.

Для выгисления $X^{r}$ воспользуемся равенством (5) при $s=r$ и леммой 3 . Применение последней возможно ввиду наличия у $B_{j}$ стандартной $\varepsilon_{i j}$-строки в случае вьполнения условия (4). При этом $\varepsilon_{i j} \in \mathbb{Z}_{m}$ является номером той $(r-1)$-ячейки, которая начинается строкой $k_{i}$, в квазистандартном $(r-1)$-блоке, начинающемся строкой $k_{j}^{*}$. Лемма 3 обеспечивает вьполнение равенства (1).

В заключение сделаем несколько замечаний. 
ЗАмЕчАниЕ 1. Условие (4), равносильное включению $\left\{k_{i}, k_{i}+1, \ldots, k_{i}+m-1\right\} \subset$ $\left\{k_{j}^{*}, k_{j}^{*}+1, \ldots, k_{j}^{*}+m^{2}-1\right\}$, может не вьполняться ни для одного $i=\overline{1, t}$ и $j=\overline{1, t}$, тогда на допустимые матрицы $B_{1}, \ldots, B_{t}$ никаких дополнительных требований не накладьвается.

ЗАмЕчАНИЕ 2. Для получения с помошью теоремы 3 простых $\partial$-графов порядка $r$ необходимо согласно следствию из теоремы 2 дополнительно обеспечить, чтобы в матрице $X$, являющейся решением уравнения (1), были две строки, неравные друг другу и неортогональные как векторы в $\mathbb{R}^{n}$. Для этого в одной из $m^{r-2}$ колонок прямоугольных ячеек на $X$ среди $m$ ненулевых прямоугольников должно быть два, не получающихся один из другого перестановкой строк.

ЗАмЕчАниЕ 3. Сформулированное в теореме 3 требование к допустимым матрицам не является необходимьм. Так при $r=2, m>2, t=1, k_{1}=p m, p \in \overline{1, m-2}$, можно взять допустимую матрицу, получающуюся из стандартной перенесением $m-m_{0}$ последних единиц ее $q$-й строки, $m_{0} \in \overline{p+1, m-1}, q \in \overline{0, p-1}$, в ее $p$-ю строку, а $m-m_{0}$ последних единиц $p$-й строки в $q$-ю. Условие (4) при $i=j=1$ справедливо, ибо

$$
\varepsilon_{11}=\frac{k_{1}-k_{1}^{*}}{m}=\frac{p m-0}{m}=p \in \mathbb{Z}_{m},
$$

но в построенной допустимой матрице нет стандартной $p$-строки, тем не менее, как легко убедиться, $X^{2}=J$.

ЗАмЕчАниЕ 4. На исчерпьвающее описание простьх $\partial$-графов в настоящее время рассчитьвать трудно. Но определенные надежды на это все же имеются в связи с тем,

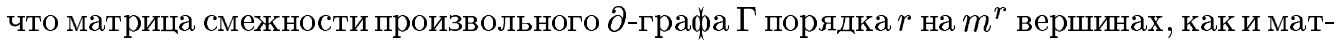
рица $A_{m^{r}}$, разбивается горизонталями на $m$ подматриц размера $m^{r-1} \times m^{r}$, у которых в каждом столбце ровно по одной единице. Для этого нумерация вершин Г выбирается так, что $m^{r-1}$ концов направленных путей длины $r$, выходящих из фиксированного начала и имеющих одну и ту же первую дугу, располагаются подряд. Для начальных значений параметров конкретно реализованньй прямой перебор позволил убедиться, что при $m=r=2$ простых $\partial$-графов нет, при $m=2, r=3$ они исчерпьваются графами $\mathscr{T}_{1}, \overline{\mathscr{T}_{1}}$ (см. рис. 1 ), а при $m=3, r=2$ - граффами $\mathscr{T}_{2}, \overline{\mathscr{T}_{2}}, \mathscr{T}_{3}, \mathscr{T}_{4}, \overline{\mathscr{T}_{4}}$ соответственно.

ЗАмЕчАниЕ 5. Среди получаемых по теореме 3 -граффов нетрудно выбрать графы, неизоморфные друг другу.

\section{СПИСОК ЦИТИРОВАННОЙ ЛИТЕРАТУРЫ}

[1] Холл М. Комбинаторика. М.: Мир, 1970.

[2] Good I. J. Normal recurring decimals // J. London Math. Soc. 1946. V. 21. P. 167-169.

[3] Fredricksen H. A survey of full length nonlinear shift register cycle algorithms // SIAM Rev. 1982. V. 24. № 2. P. 195-221.

[4] Кратко М. И., Строк В. В. Последовательности де Брейна с ограничениями // Вопросы кибернетики. Комбинаторный анализ и теория графов. М.: Наука, 1980. С. 80-84.

[5] Ширишева И. В. О числе конечных автоматов, устанавливаемых постоянным входом в фиксированное состояние // Дискретная матем. 1994. Т. 6. № 4. С. 80-86.

[6] Харари $\Phi$. Теория графов. М.: Мир, 1973.

[7] Allow C. M., Brenner J. L. Roots and canonical forms for circulant matrices // Trans. Amer. Math. Soc. 1963. V. 107. P. 60-76. 